\title{
A CHARACTERIZATION OF SMOOTH CANTOR BOUQUETS
}

\author{
WITOLD D. BULA AND LEX G. OVERSTEEGEN
}

(Communicated by James E. West)

\begin{abstract}
We prove that all smooth fans having a dense set of endpoints are topologically equivalent.
\end{abstract}

Let $X$ be a smooth fan whose set of endpoints is dense in $X$. Such fans have been constructed, e.g., by J. H. Roberts [6], who proved that the space of rational sequences of the Hilbert cube can be embedded in the Cantor fan, and by A. Lelek [3], who showed the existence of a fan whose (one-dimensional) set of endpoints can be connectified by adding the vertex. Lately, spaces similar to $X \backslash\{v\}$, where $v$ is the vertex of $X$, were discovered to be Julia sets of some nice analytic functions (see R. L. Devaney and M. Krych [2]; see also J. C. Mayer [4]). We are going to prove that all such examples are homeomorphic.

Theorem. All smooth fans having dense set of endpoints are topologically equivalent.

Let us recall that a continuum $X$ is said to be hereditarily unicoherent if $K \cap L$ is connected for every pair $K, L$ of subcontinua of $X$. A continuum $X$ is called a dendroid if it is arcwise connected and hereditarily unicoherent. By a fan we will mean a dendroid having exactly one ramification point; we will call this point the vertex of $X$. A fan $X$ is said to be smooth if the sequence of arcs $\left[v, x_{n}\right]$ converges to the arc $[v, x]$ for every sequence $x_{n}$ converging to $x$, where $x, x_{n} \in X$ and $v$ is the vertex of $X$. If $X$ is a fan, then $E(X)$ will denote the set of endpoints of $X$. If $x, y \in \mathfrak{R} \times \mathfrak{R}$, then by $|x-y|$ we will denote the Euclidean distance between points $x$ and $y$ and by $[x, y]$ we will mean the linear segment with endpoints $x$ and $y$.

\section{MAPPINGS BETWEEN INVERSE SYSTEMS}

The following lemma is similar to $\left[5\right.$, Theorem $\left.2^{\prime}\right]$. The proof is a standard inductive argument and is left to the reader.

Received by the editors October 18, 1988.

1980 Mathematics Subject Classification (1985 Revision). Primary 54F20, 54F65.

Key words and phrases. Fan, smooth fan.

The first author was supported in part by NSERC grant number A5616, and the second author was supported in part by NSF-DMS- 8602400 and NSF-Alabama grant number RII-8610669. 
Lemma 1. Suppose $P_{n}$ and $Q_{n}$ are compact metric spaces, $X=\lim _{\longleftarrow}\left\langle P_{n}, p_{m}^{n}\right\rangle$, $Q=\lim _{\longleftarrow}\left\langle Q_{n}, q_{m}^{n}\right\rangle$, and $\mathscr{F}_{n}$ is a class of mappings from $P_{n}$ onto $Q_{n}$ such that for every $n$, positive real $\varepsilon$, and mapping $f \in \mathscr{F}_{n}$, there exists a mapping $g \in$ $\mathscr{F}_{n+1}$ such that the following diagram is $\varepsilon$-commutative, i.e. $\operatorname{dist}\left(g \circ p_{n}^{n+1}(x)\right.$, $\left.q_{n}^{n+1} \circ g(x)\right)<\varepsilon$ for each $x \in P_{n+1}$.

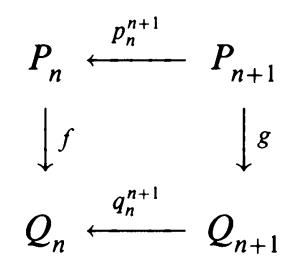

Let $\varepsilon_{n}$ be a decreasing sequence converging to 0 . Then for each $n$ there exists an $h_{n} \in \mathscr{F}_{n}$ such that the following diagram is $\varepsilon_{n}$-commutative for every $k \leq n \leq m$.

$(*)$

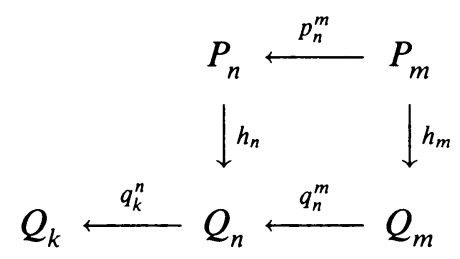

By Mioduszewski's result [5, Theorem 2], the sequence $h_{n}$ induces a map $h: X \stackrel{\text { onto }}{\longrightarrow} Y$ defined by $h\left(\left(x_{1}, x_{2}, \ldots\right)\right)=\left(y_{1}, y_{2}, \ldots\right)$, where

$$
y_{n}=\lim _{m \rightarrow \infty} q_{n}^{m} \circ h_{m}\left(x_{n}\right) .
$$

Let us omit the standard proof of the following lemma.

Lemma 2. Let $X=\lim _{\longleftarrow}\left\langle P_{n}, p_{m}^{n}\right\rangle, \quad Y=\lim _{\lfloor}\left\langle Q_{n}, q_{m}^{n}\right\rangle, \quad\left\{\varepsilon_{n}: n=1,2, \ldots\right\}$, $\left\{h_{n}: n=1,2, \ldots\right\}$, and $h$ be as in the statement of Lemma 1. Assume, in addition, that spaces $X, P_{n}$, and $Q_{n}$ are embedded in the Hilbert cube in such a way that $p_{n}: X \rightarrow P_{n}$ and $q_{n}: Y \rightarrow Q_{n}$ are $1 / n$-mappings. Let $x_{n} \in P_{n}$ be a sequence converging to $x \in X$. Then $\lim _{n \rightarrow \infty} h_{n}\left(x_{n}\right)=h(x)$.

\section{PreliminaRY LeMMAS}

Let $C$ denote a Cantor set lying in $[0,1] \times\{1\} \subseteq \mathfrak{R} \times \mathfrak{R}$. Let $v=\langle 1 / 2,0\rangle$ and let $T$ be the Cantor fan being the union of all linear segments joining $v$ with points of $C$. Let $\rho: T \rightarrow[0,1]$ be the natural second coordinate projection. Let $X$ be a fan such that $E(X)$ is dense in $X$. By the result of Carruth [1], we may assume that $X$ is embedded in $T$. There is a natural monotone mapping $\pi: X \backslash\{v\} \rightarrow C$ such that $e \in[v, \pi(e)]$ for every $e \in E(X)$. We may assume that $\pi(E(X))$ is dense in $C$. The assertion of the following lemma is a consequence of the density of $E(X)$. 
Lemma 3. For every point $e \in E(X)$ there is a sequence $\left\{e_{n} \in E(X): n=\right.$ $1,2, \ldots\}$ such that $\pi(e)=\lim _{n \rightarrow \infty} \pi\left(e_{n}\right)$ and $\operatorname{cl}\left\{e_{n}: n=1,2, \ldots\right\}=[v, e] \cup$ $\left\{e_{n}: n=1,2, \ldots\right\}$.

For a subset $A$ of $C$ define

$$
h(A)=\sup \{\rho(e): e \in E(X) \text { and } \pi(e) \in A\} .
$$

Without loss of generality we may assume that $h(C)=1$. Let us omit the easy proof of the following lemma.

Lemma 4. For every nonempty closed-and-open subset $U$ of $C$ there is a point $e \in E(X)$ such that $\pi(e) \in U$ and $\rho(e)=h(U)$.

Lemma 5. For every $\varepsilon>0$, nonempty closed-and-open subset $U$ of $C$ and point $e \in E(X)$ such that $\pi(e) \in U$ and $\rho(e)=h(U)$, there is a null partition $\left\{U_{n}: n=1,2, \ldots\right\}$ of $U \backslash\{\pi(e)\}$ into closed-and-open subsets of $C$ of diameter less than $\varepsilon$ such that $\left\{h\left(U_{n}\right): n=1,2, \ldots\right\}$ is dense in $\rho([v, e])$. Moreover, if $e_{n} \in E(X)$ is such that $\pi\left(e_{n}\right) \in U_{n}$ and $\rho\left(e_{n}\right)=h\left(U_{n}\right)$, then $\operatorname{cl}\left\{e_{n}: n=\right.$ $1,2, \ldots\}=[v, e] \cup\left\{e_{n}: n=1,2, \ldots\right\}$.

Proof. By Lemma 3, there is a sequence $f_{m} \in E(X)$ such that $\pi(e)=$ $\lim _{m \rightarrow \infty} \pi\left(f_{m}\right)$ and $\operatorname{cl}\left\{f_{m}: m=1,2, \ldots\right\}=[v, e] \cup\left\{f_{m}: m=1,2, \ldots\right\}$. Let $\mathscr{T}=\left\{W_{m}: m=1,2, \ldots\right\}$ be a sequence of disjoint closed-and-open subsets of $U \backslash\{\pi(e)\}$ such that $\pi\left(f_{m}\right) \in W_{m}$, $\operatorname{diam} W_{m}<\varepsilon, \lim _{m \rightarrow \infty} \operatorname{diam} W_{m}=0$, and $\left|\rho\left(f_{m}\right)-h\left(W_{m}\right)\right|<1 / m$. Observe that $\left\{h\left(W_{m}\right): m=1,2, \ldots\right\}$ is dense in $\rho([v, e])$. Hence, any completion of $\mathscr{T}$ to a null partition of $U \backslash\{\pi(e)\}$ with mesh less than $\varepsilon$ will satisfy the assertion of the lemma.

\section{Construction of an INVERSE SeQuence $\left\langle T_{n}, p_{m}^{n}\right\rangle$ Associated with $X$}

Choose $e_{0} \in E(X)$ such that $\rho\left(e_{0}\right)=h(C)$. Let $T_{0}=\left[v, e_{0}\right]$ and let $p_{0}: X \rightarrow T_{0}$ be the horizontal projection onto $T_{0}$. Since $\rho\left(e_{0}\right)=h(C)$, the map $p_{0}$ is well defined. We will call $e_{0}$ the endpoint of $T_{0}$ and will write $E\left(T_{0}\right)=\left\{e_{0}\right\}$. Put $\mathscr{T}_{0}=\{C\}$ and let $\mathscr{T}_{1}=\left\{U_{n}: n=1,2, \ldots\right\}$ be a partition of $C \backslash\left\{\pi\left(e_{0}\right)\right\}$ guaranteed by Lemma 5 for $e=e_{0}$ and $\varepsilon=1$. For every $n$ choose $e_{n} \in E(X)$ such that $\pi\left(e_{n}\right) \in U_{n}$ and $\rho\left(e_{n}\right)=h\left(U_{n}\right)$. Let $T_{1}=$ $\left.T_{0} \cup \bigcup\left\{\mid v, e_{n}\right]: n=1,2, \ldots\right\}$. We may define $p_{1}: X \rightarrow T_{1}$ in such a way that $p_{1} \mid T_{0}$ is the identity and $p_{1} \mid \pi^{-1}\left(U_{n}\right)$ is the horizontal projection into $\left[v, e_{n}\right]$. Suppose we have already defined sets $T_{0}, \ldots, T_{n} \subset X$, mappings $p_{k}: X \rightarrow T_{k}$ for $k=0, \ldots, n$, and collections $\mathscr{T}_{0}, \ldots, \mathscr{T}_{n}$ such that

(1) $T_{k}$ is a fan for $k=1,2, \ldots, n$,

(2) $T_{0} \subset T_{1} \subset \cdots \subset T_{n}$,

(3) $E\left(T_{0}\right) \subset E\left(T_{1}\right) \subset \cdots \subset E\left(T_{n}\right)$,

(4) $p_{k+1} \mid T_{k}$ is the identity for $k=0, \ldots, n-1$,

(5) $\mathscr{\mathscr { T }}_{k+1}$ is a null family of disjoint closed-and-open sets of diameter less than or equal to $1 /(k+1)$, refining $\mathscr{T}_{k}$ and such that $\cup \mathscr{T}_{k+1}=C \backslash$ $\pi\left(E\left(T_{k}\right)\right)$, 
(6) for every $U \in \mathscr{T}_{k}$ there is a unique point $e_{k}(U) \in E\left(T_{k}\right)$ such that $\pi\left(e_{k}(U)\right) \in U$; further,

(a) $\rho\left(e_{k}(U)\right)=h(U)$,

(b) $p_{k} \mid \pi^{-1}(U)$ is the horizontal projection into $\left[v, e_{k}(U)\right]$ and

(c) if $W \in \mathscr{T}_{k-1}$ and $\mathscr{T}=\left\{U \in \mathscr{T}_{k}: U \subset W\right\}$, then

$$
\operatorname{cl}\left\{e_{k}(U): U \in \mathscr{T}\right\}=\left\{e_{k}(U): U \in \mathscr{T}\right\} \cup\left[v, e_{k-1}(W)\right] .
$$

Observe that in view of Lemmas 4 and 5 the induction can be continued. Define $p_{m}^{n}=p_{m} \mid T_{n}$ for $m \leq n$ and observe that $p_{m}=p_{m}^{n} \circ p_{n}$ and $\rho(x)=$ $\rho \circ p_{n}(x)$ for every $n$ and $x \in X$. Since $X=\operatorname{cl} \bigcup\left\{T_{n}: n=0,1, \ldots\right\}$ and every $p_{n}: X \rightarrow T_{n}$ is a $1 / n$-map, the space $X$ is homeomorphic to $\lim _{\longleftarrow}\left\langle T_{n}, p_{m}^{n}\right\rangle$ and the maps $p_{n}$ converge to the identity on $X$. We will say that the above inverse sequence is associated with the fan $X$.

\section{CONSTRUCTION OF A HOMEOMORPHISM}

Now, let $X$ and $Y$ be smooth fans having a dense set of endpoints and let $\left\langle P_{n}, p_{m}^{n}\right\rangle$ and $\left\langle Q_{n}, q_{m}^{n}\right\rangle$ be inverse sequences associated with $X$ and $Y$, respectively. To complete the proof of the theorem we will construct a sequence of homeomorphisms $h_{n}: P_{n} \stackrel{\text { onto }}{\rightarrow} Q_{n}$ inducing a homeomorphism between the limit spaces.

Let $v$ be the vertex of $X$ and $w$ the vertex of $Y$. We may assume that $\rho(X)=\rho(Y)=[0,1]$. Let $h_{0}: P_{0} \stackrel{\text { onto }}{\rightarrow} Q_{0}$ be the linear map such that $h_{0}(v)=$ $w$. Let $\mathscr{F}_{0}=\left\{h_{0}\right\}$. Let $\left\{e_{n}: n=1,2, \ldots\right\}=E\left(P_{1}\right) \backslash E\left(P_{0}\right)$ and $\left\{f_{n}: n=\right.$ $1,2, \ldots\}=E\left(Q_{1}\right) \backslash E\left(Q_{0}\right)$. We may find a permutation $\varphi$ of positive integers such that

$$
\left|\rho\left(e_{n}\right)-\rho\left(f_{\varphi(n)}\right)\right| \leq \min \left\{\frac{1}{\alpha(n) \sqrt{5}}, \frac{\rho\left(e_{n}\right)}{4}\right\}
$$

for every $n$, where $\alpha(n)=\min \{n, \varphi(n)\}$.

Let $h_{1}: P_{1} \stackrel{\text { onto }}{\rightarrow} Q_{1}$ be the extension of $h_{0}$, such that $h_{1}$ maps $\left[v, e_{n}\right.$ ] linearly onto $\left[w, f_{\varphi(n)}\right.$ ] for every $n$. Observe that if $x \in P_{1} \backslash\{v\}$, then $\pi \circ q_{0}^{1} \circ h_{1}(x)=$ $\pi \circ h_{0} \circ p_{0}^{1}(x)$. Hence, $\left|q_{0}^{1} \circ h_{1}(z)-h_{0} \circ p_{0}^{1}(z)\right| \leq 1 / 2$ for every $z$.

For each nonnegative integer $n$ we will inductively construct a class $\mathscr{F}_{n}$ of homeomorphisms mapping $P_{n}$ onto $Q_{n}$ such that for every $h \in \mathscr{F}_{n}$ and $\varepsilon>0$ there is a $g \in \mathscr{F}_{n+1}$ satisfying the following conditions:

(7) $g \mid P_{n}=h$,

(8) for every $e \in E\left(P_{n}\right)$ the function $h \mid[v, e]:[v, e] \stackrel{\text { onto }}{\rightarrow}[w, h(e)]$ is a linear homeomorphism,

(9) $\pi \circ q_{n}^{n+1} \circ g(x)=\pi \circ h \circ p_{n}^{n+1}(x)$,

(10) $\left|\rho \circ q_{n}^{n+1} \circ q(x)-\rho \circ h \circ p_{n}^{n+1}(x)\right|<\varepsilon$, and

(11) $|\rho(x)-\rho \circ h(x)|<\rho(x) / 4$ for each $x \in P_{n}$.

Suppose that classes of homeomorphisms $\mathscr{F}_{i}$ satisfying $(7)-(11)$ have already been defined for each $i, 0 \leq i \leq n$. Let $\mathscr{U}_{i}\left[\mathscr{V}_{i}\right]$ be the partition 
of $C \backslash E\left(P_{i-1}\right)$ [of $C \backslash E\left(Q_{i-1}\right)$, respectively] used for the construction of $P_{i}\left[Q_{i}\right]$, where $i=1,2, \ldots, n$. Let $\left\{U_{k}: k=1,2, \ldots\right\}$ be an enumeration of $\mathscr{U}_{n}$ and let $e_{k}=e\left(U_{k}\right)$. The homeomorphism $h: P_{n} \rightarrow Q_{n}$ maps each $e_{k}$ to a point $f_{k} \in E\left(Q_{n}\right)$. Let $V_{k}$ be the unique element in $\mathscr{V}_{n}$ containing $f_{k}$. Then $\left\{V_{k}: k=1,2, \ldots\right\}$ is an enumeration of $\mathscr{V}_{n}$. For every $k$, let $\mathscr{U}_{n+1}\left(U_{k}\right)=\left\{U \in \mathscr{U}_{n+1}: U \subset U_{k}\right\}$, and $\mathscr{V}_{n+1}\left(V_{k}\right)=\left\{V \in \mathscr{V}_{n+1}: V \subset V_{k}\right\}$. Let $\left\{U_{k, j}: j=1,2, \ldots\right\}$ and $\left\{V_{k, j}: j=1,2, \ldots\right\}$ be enumerations of $\mathscr{U}_{n+1}\left(U_{k}\right)$ and $\mathscr{V}_{n+1}\left(V_{k}\right)$, respectively. Put $e_{k, j}=e\left(U_{k, j}\right)$ and $f_{k, j}=f\left(V_{k, j}\right)$. Recall that $\rho(x)=\rho \circ p_{m}^{n}(x)$. By (11), $\left|\rho\left(e_{k, j}\right)-\rho \circ h \circ p_{n}^{n+1}\left(e_{k, j}\right)\right|<\rho\left(e_{k, j}\right) / 4$.

For every $k$ we may find a permutation $\varphi_{k}$ of positive integers such that

$$
\left|\rho\left(e_{k, j}\right)-\rho\left(f_{k, \varphi_{k}(j)}\right)\right|<\frac{\rho\left(e_{k, j}\right)}{4}
$$

and

$$
(* * *) \quad\left|\rho \circ h \circ p_{n}^{n+1}\left(e_{k, j}\right)-\rho\left(f_{k, \varphi_{k}(j)}\right)\right|<\min \left\{\varepsilon, \frac{1}{\alpha(j)+k}\right\},
$$

where $\alpha(j)=\min \left\{j, \varphi_{k}(j)\right\}$. Let $g$ be the extension of $h$ which maps $\left[v, e_{k, j}\right]$ linearly onto $\left[w, f_{k, \varphi_{k}(j)}\right]$. Then (7) and (8) follow immediately; (10) and the continuity of $g$ follow from $(* * *)$ and the linearity of $g$; and (11) for $g$ follows from (11) for $h$, the condition $(* *)$, and the fact that $h \mid\left[v, e_{k, j}\right]$ is linear.

Let $\mathscr{F}_{n+1}$ be the class of homeomorphisms $g$ mapping $P_{n+1}$ onto $Q_{n+1}$ obtained as described above for every $h \in \mathscr{F}_{n}$ and $\varepsilon=1 / r$, where $r=1,2, \ldots$.

By Lemma 1, we can select a sequence $h_{n} \in \mathscr{F}_{n}$ of homeomorphisms such that for each $k \leq n \leq m$ diagram $(*)$ is $1 / 2^{n}$-commutative. Hence the homeomorphisms $h_{n}$ induce a continuous map $h: X \stackrel{\text { onto }}{\longrightarrow} Y$ defined by $h(x)=y$, where $q_{s}(y)=\lim _{n \rightarrow \infty} q_{s}^{n} \circ h_{n} \circ p_{n}(x)$. To complete the proof it suffices to show that $h$ is one-to-one.

Let $x \neq a \in X$ and let $x_{n}=p_{n}(x), a_{n}=p_{n}(a), h(x)=y$, and $h(a)=b$. Suppose first that for some $n, \pi\left(x_{n}\right) \neq \pi\left(a_{n}\right)$. By (9), the definition of $h$ and the fact that each $h_{n}$ is a homeomorphism, $\pi \circ q_{n} \circ h(x) \neq \pi \circ q_{n} \circ h(a)$, and $h(x) \neq h(a)$. Hence we may assume that either $\pi\left(x_{n}\right)=\pi\left(a_{n}\right)$, for each $n$ or $a=v$.

Then there exists a (unique) $e \in E\left(P_{n}\right)$ such that $p_{n}([v, e]) \subset\left[v, e_{n}\right]$. Since $e \in E(X), \operatorname{Lim}_{n \rightarrow \infty}\left[v, e_{n}\right]=[v, e]$ and $\lim _{n \rightarrow \infty} \rho\left(e_{n}\right)=\rho(e)$. Clearly, $\rho\left(e_{n+1}\right) \leq \rho\left(e_{n}\right)$ for each $n$. By (11),

$$
\rho \circ h_{n}\left(e_{n}\right)>\frac{3}{4} \rho\left(e_{n}\right) \geq \frac{3}{4} \rho(e)
$$

for each $n$. Since each $h_{n}$ is linear,

$$
\left|\rho \circ h_{n}\left(x_{n}\right)-\rho \circ h_{n}\left(y_{n}\right)\right| \geq \frac{3}{4}\left|\rho\left(x_{n}\right)-\rho\left(y_{n}\right)\right|
$$


for each $n$ and $x_{n}, y_{n} \in\left[v, e_{n}\right]$. Since $\operatorname{Lim}_{n \rightarrow \infty}\left[v, e_{n}\right]=[v, e]$, there exist $c_{n}, d_{n} \in\left[v, e_{n}\right]$ such that $\lim _{n \rightarrow \infty} c_{n}=x$ and $\lim _{n \rightarrow \infty} d_{n}=a$. Hence, $\lim _{n \rightarrow \infty}\left|\rho \circ h_{n}\left(c_{n}\right)-\rho \circ h_{n}\left(d_{n}\right)\right| \geq \lim _{n \rightarrow \infty}\left|\rho\left(c_{n}\right)-\rho\left(d_{n}\right)\right|=|\rho(x)-\rho(a)|>0$.

Hence, by Lemma 2, $|\rho \circ h(x)-\rho \circ h(a)|>0$ and $h(x) \neq h(a)$.

Added in proof. The main result of this paper was proved independently by $\mathrm{W}$. J. Charatonik [The Lelek fan is unique, (to appear in Houston J. of Math.)].

\section{REFERENCES}

1. J. H. Carruth, A note on partially ordered compacta, Pacific J. Math. 24 (1968), 229-231.

2. R. L. Devaney and M. Krych, Dynamics of $\exp (z)$, Ergodic Theory and Dynamical Systems 4 (1984), 35-52.

3. A. Lelek, On plane dendroids and their end points in the classical sense, Fund. Math. 49 (1961), 301-319.

4. J. C. Mayer, An explosion point for the set of endpoints of the Julia set of $\lambda \exp (z)$, (to appear in Ergodic Theory and Dynamical Systems).

5. J. Mioduszewski, Mappings of inverse limits, Colloq. Math. 10 (1963), 39-44.

6. J. H. Roberts, The rational points in Hilbert space, Duke Math. J. 23 (1956), 489-491.

University of Saskatchewan, Saskatoon S7N 0W0 Canada

University of Alabama at Birmingham, Birmingham, Alabama 35294 